\title{
RECENT ADVANCES IN HYDROGELS FOR CARTILAGE TISSUE ENGINEERING
}

\author{
S.L. Vega, M.Y. Kwon and J.A. Burdick*
}

Department of Bioengineering, University of Pennsylvania, Philadelphia, PA, 19104, USA

\begin{abstract}
Articular cartilage is a load-bearing tissue that lines the surface of bones in diarthrodial joints. Unfortunately, this avascular tissue has a limited capacity for intrinsic repair. Treatment options for articular cartilage defects include microfracture and arthroplasty; however, these strategies fail to generate tissue that adequately restores damaged cartilage. Limitations of current treatments for cartilage defects have prompted the field of cartilage tissue engineering, which seeks to integrate engineering and biological principles to promote the growth of new cartilage to replace damaged tissue. To date, a wide range of scaffolds and cell sources have emerged with a focus on recapitulating the microenvironments present during development or in adult tissue, in order to induce the formation of cartilaginous constructs with biochemical and mechanical properties of native tissue. Hydrogels have emerged as a promising scaffold due to the wide range of possible properties and the ability to entrap cells within the material. Towards improving cartilage repair, hydrogel design has advanced in recent years to improve their utility. Some of these advances include the development of improved network crosslinking (e.g. double-networks), new techniques to process hydrogels (e.g. 3D printing) and better incorporation of biological signals (e.g. controlled release). This review summarises these innovative approaches to engineer hydrogels towards cartilage repair, with an eye towards eventual clinical translation.
\end{abstract}

Keywords: Hydrogel, biomaterial, cartilage, tissue engineering.

*Address for correspondence:

J.A. Burdick

Department of Bioengineering

University of Pennsylvania

Philadelphia, PA, 19104, USA

Email: burdick2@seas.upenn.edu

\section{Introduction}

In simplistic terms, articular cartilage is a tissue consisting of a single cell type (chondrocyte) embedded within an extracellular matrix (ECM). However, the structure is more complex and includes three depth-dependent layers: the superficial zone, the middle zone and the deep zone, with changes in ECM content, structure and chondrocyte behaviour with depth. In the middle zone - the largest region - cartilage tissue is stiff, avascular, and features a low density of rounded chondrocytes surrounded by an ECM consisting of glycosaminoglycans (GAGs) and type II collagen (Vunjak-Novakovic and Freed, 1998; Wong and Carter, 2003). Cartilage damage due to trauma typically begins as a focal defect, which later progresses to a full-thickness defect once the lesion comes into contact with surrounding bone. Due to low cellularity, low vascularisation, minimal proliferative capacity of residing chondrocytes and low cell migration to areas of damage, articular cartilage is intrinsically unable to repair itself (Ahmed and Hincke, 2010).

Current strategies to repair focal and full-thickness cartilage defects have evolved from surgery aimed at inducing endogenous repair mechanisms (e.g. microfracture), towards osteochondral transplantation, and more recently to cell-based repair techniques, such as autologous chondrocyte implantation (ACI) (Brittberg, 2008; Goldring, 2006; Hunziker, 2002). ACI is a procedure in which patient chondrocytes are implanted into a debrided cartilage lesion (Peterson et al., 2010). Both microfracture and ACI lack 3D scaffolds to define where cells reside and to instruct matrix formation; however, there have been advances in these therapies in recent years. Matrixinduced autologous chondrocyte implantation (MACI) is a cell-based therapy that supplements ACI by providing chondrocytes with a supportive scaffold material for matrix formation (Makris et al., 2015). Autologous matrixinduced chondrogenesis (AMIC), on the other hand, supplements microfracture with an acellular scaffold in the lesion as an attempt to increase mechanical stability at the site, cell migration and cartilage production (Benthien and Behrens, 2011). Despite promising results, these clinical techniques are not without their limitations, including the surgical procedures and the quality of the formed cartilage.

Towards further expanding the use of biomaterials in cartilage repair, hydrogels have gained a great deal of interest in cartilage tissue engineering (Balakrishnan and Banerjee, 2011). Hydrogels are 3-dimensional (3D) polymer networks that are highly swollen and porous on the molecular scale, allowing the diffusion of various solutes and nutrients. Their fabrication can be cell amenable, allowing the encapsulation of different cell types (e.g. chondrocytes, stem cells). Lastly, an assortment of 
hydrogel parameters can also be tuned, including polymer chemistry, crosslinking density, degradation, mechanical properties and release kinetics of biochemical factors, towards improving their utility in tissue repair (Spiller et al., 2011). Numerous studies have characterised the effects of hydrogel crosslinking - tuned through parameters like macromer concentration - on neocartilage formation (Chung et al., 2006). These studies have shown that matrix distribution can be hindered when a hydrogel with a high degree of crosslinking is used. Also, differences in the composition of hydrogels have been investigated in the context of cartilage formation. For example, several groups report that type II collagen hydrogels induce a higher amount of chondrogenic outputs, in comparison to type I collagen hydrogels with similar properties ( $\mathrm{Lu}$ et al., 2010; Nehrer et al., 1997).

To serve as constructs to replace articular cartilage, hydrogels have been used as either acellular scaffolds or as cell-laden biomaterials (Spiller et al., 2011). For both strategies, hydrogel implants must integrate with surrounding tissue and support the production of cartilage. Acellular constructs are almost exclusively assayed in vivo, where the goal is structural and mechanical properties similar to native cartilage, while allowing efficient load transfer, or the recruitment of cells for cartilage repair (Kobayashi and Oka, 2004; Stammen et al., 2001). Cell-laden hydrogels present residing cells with either developmental cues to trigger chondrogenesis, or microenvironmental cues that mimic native cartilage to maintain a chondrogenic phenotype and encourage matrix formation. Hydrogels are being developed to present these signals either as molecules bound to the polymer or through their controlled release. Also, these cultures may be performed in vitro, such as in bioreactors, or may be implanted directly for tissue growth.

With rapid advances being made towards hydrogels for cartilage tissue engineering, the goal of this review is to cover current and emerging developments in hydrogel design for cartilage repair. It is not meant to be comprehensive, but rather highlight representative advances in the field in recent years with a particular focus on hydrogel design. Specific areas include the use of unique crosslinking to produce hydrogels with strong mechanical properties, the improved processing of hydrogels into macroporous structures and the incorporation of biological signals to improve cell behaviour.

\section{Improvements in hydrogel structure}

One of the key design criteria for hydrogels towards cartilage regeneration is mechanical integrity. Traditional hydrogel designs, based on networks of a single polymer, generally result in hydrogel constructs with mechanical properties far inferior to those of native cartilage. Also, the increase in hydrogel modulus through increased crosslink density may compromise the viability of encapsulated cells through reduced diffusion. With the aim of increasing the mechanical properties of hydrogels to approach those of hyaline cartilage, the focus is shifting from conventional hydrogels that use a single polymer for hydrogel fabrication
(Fig. 1a), to more complex hydrogel systems with mixtures of multiple polymers, often including two or more independent networks. These systems not only typically achieve stronger mechanical properties than networks of single polymers, but may exhibit superior integration with surrounding tissue in vivo. In this section, we examine recent advances within the framework of different network types (Fig. 1).

\section{Interpenetrating networks}

Hydrogels based on interpenetrating networks (IPNs) are comprised of two or more separate crosslinked networks not covalently bound to each other, but rather partially intertwined such that chemical bonds have to be broken to separate the components that form the networks (Fig. 1b). Consequentially, the mechanical properties of IPN hydrogels tend to be stronger than those created with individual component networks, which makes them appealing for cartilage tissue engineering applications. In recent years, the design of IPNs for hydrogel formation has turned to the incorporation of two or more additional networks to either better mimic the physical properties of native tissue or to recapitulate the presentation of bioactive cues available to cells in the hydrogel constructs.

Recent examples of IPNs include the work done by Ingavle and colleagues, in which they explored the incorporation of methacrylated chondroitin sulphate, a major component of the cartilage ECM, as the second network after diffusion into an existing agarosepoly(ethylene glycol) diacrylate (PEGDA) network. Incorporating chondroitin sulphate into the network significantly increased the viability of encapsulated chondrocytes for at least six weeks and promoted greater biosynthesis of collagen and GAGs in the pericellular matrix (Ingavle et al., 2013). Reinforcing networks such as those provided by methacrylated hyaluronic acid (HA) within a fibrin hydrogel have been established as well (Fig. 2a) (Snyder et al., 2014). Snyder and colleagues found that the incorporation of a crosslinked HA network increased the compressive moduli of hydrogel constructs and modulated gene expression of encapsulated human mesenchymal stem cells (MSCs) by decreasing expression of the dedifferentiation marker type I collagen and increasing expression of the chondrogenic transcription factor Sox9 (Snyder et al., 2014).

In addition to IPNs of two networks, several groups have also investigated how tri-component IPNs increase mechanical properties and cartilage formation using both non-cell instructive (Dinescu et al., 2015; Liao et al., 2013) and cell-instructive materials (Guo et al., 2012). In a noncell instructive scaffold, comprised of gelatin, alginate and polyacrylamide, Dinescu and co-workers reported higher cell proliferation, lower cytotoxicity and greater chondrogenic gene expression (Sox9, type II collagen) of human adipose-derived stem cells than in one- or two-component hydrogels. The authors attributed these findings to the tri-component network's ability to better retain its internal structure and porosity in long-term culture (Dinescu et al., 2015). Guo et al. (2012) further used this tri-component IPN concept to better approximate the diversity of biomaterials present in articular cartilage. They 
(a)

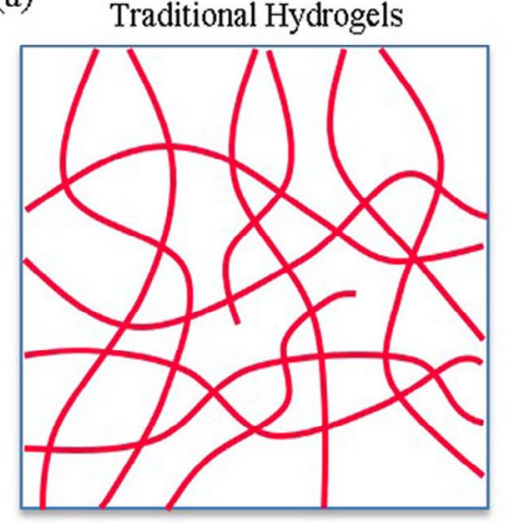

(d)
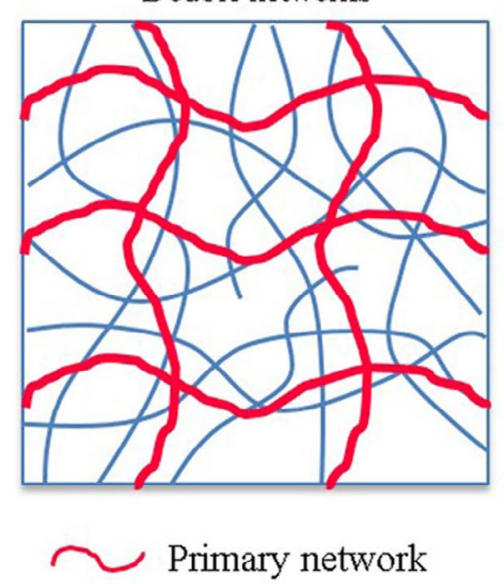

$\sim$ Secondary network (b)

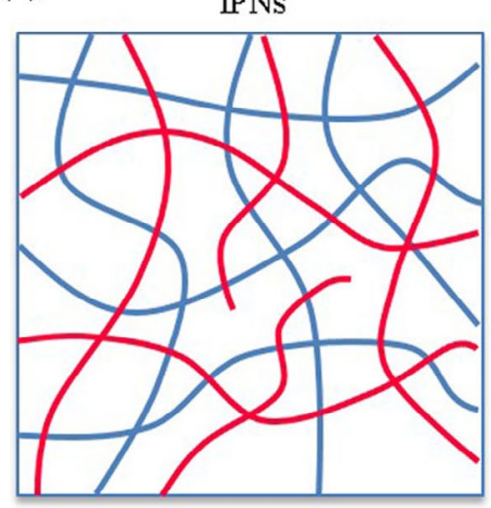

(e)

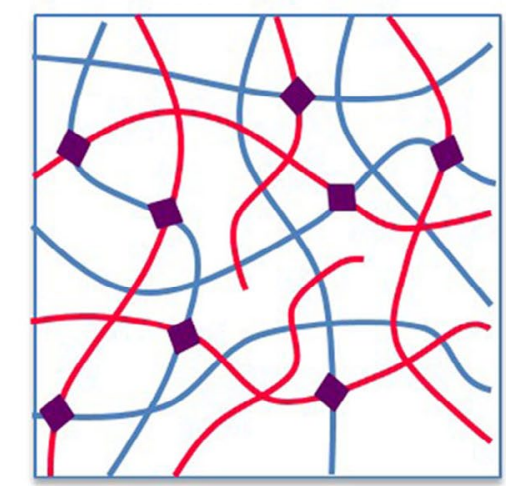

Secondary polymer

Covalent crosslink (c)

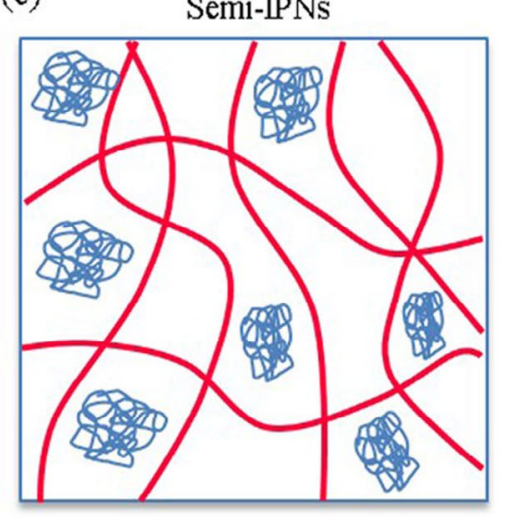

(f)

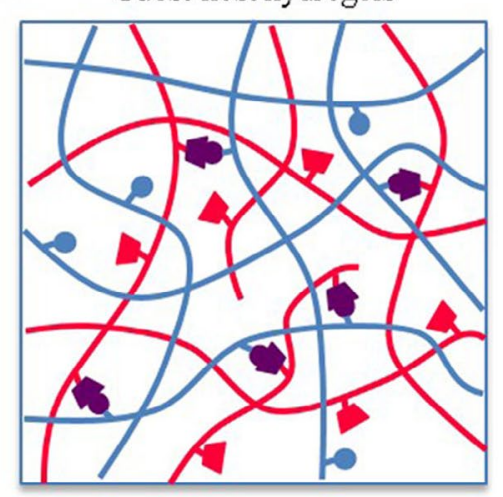

toy Guest-host interaction

Fig. 1. Schematic representation of different designs utilised in hydrogels, from (a) traditional single polymer networks to those that include (b,c) multiple networks and (d-f) mixtures of polymers. Double networks may be linked together, but this is not a requirement. Generally, the network design controls properties such as mechanics and degradation.

demonstrated the feasibility of generating a tri-component IPN using only cell-instructive components (collagen combined with methacrylate-modified chondroitin sulphate and HA) (Guo et al., 2012). By comparing it to a semi-IPN using the same components (where the chondroitin sulphate and HA were not methacrylated) and by varying the extent of methacrylation, they demonstrated tuneable mechanics with the tri-component IPNs, increased gene expression of chondrogenic markers (i.e. aggrecan, type II collagen and Sox9), downregulation of the dedifferentiation marker type I collagen and increased collagen and GAG synthesis by encapsulated rabbit chondrocytes that correlated with the bulk mechanics of the IPN constructs (Guo et al., 2012).

\section{Semi-interpenetrating networks}

Unlike IPNs, semi-IPNs consist of a crosslinked network with either linear or branched polymers entrenched within the network (Fig. 1c). As such, the polymers and the network can theoretically be separated from one another without breaking chemical bonds. One of the most common macromolecules distributed in these networks is HA. In a recent study, high molecular weight HA was distributed within injectable networks of photopolymerised methacrylated chitosan (MeGC) (Park et al., 2013). An optimum formulation and gelation protocol was developed, and the presence of entangled (but unbound) HA (350 kDa) incorporated into the chitosan network resulted in stronger proteoglycan and GAG staining (as measured by alcian blue and safranin O) in the lacunae of chondrocytes encapsulated and cultured for up to $21 \mathrm{~d}$ (Park et al., 2013).

Another approach towards utilising the properties of semi-IPNs is exploiting their ability to leach low-molecular weight macromolecules over time, as demonstrated by Skaalure et al. in a degradable poly(ethylene glycol) (PEG) hydrogel with low-molecular weight HA (29 kDa) (Skaalure et al., 2014). By comparing PEG networks infiltrated by either low- or high-molecular weight HA (2 MDa), the authors found that leaching of low molecular weight HA over a $28 \mathrm{~d}$ period led to the greatest soluble GAG deposition during construct maturation, while collagen biosynthesis was comparable to that generated in constructs with high molecular weight HA (which remained in the network during this period) (Skaalure et al., 2014). Conceptually similar approaches taken by Little et al. suggest that low-molecular weight HA and chondroitin sulphate in a fibrin-alginate hydrogel may 

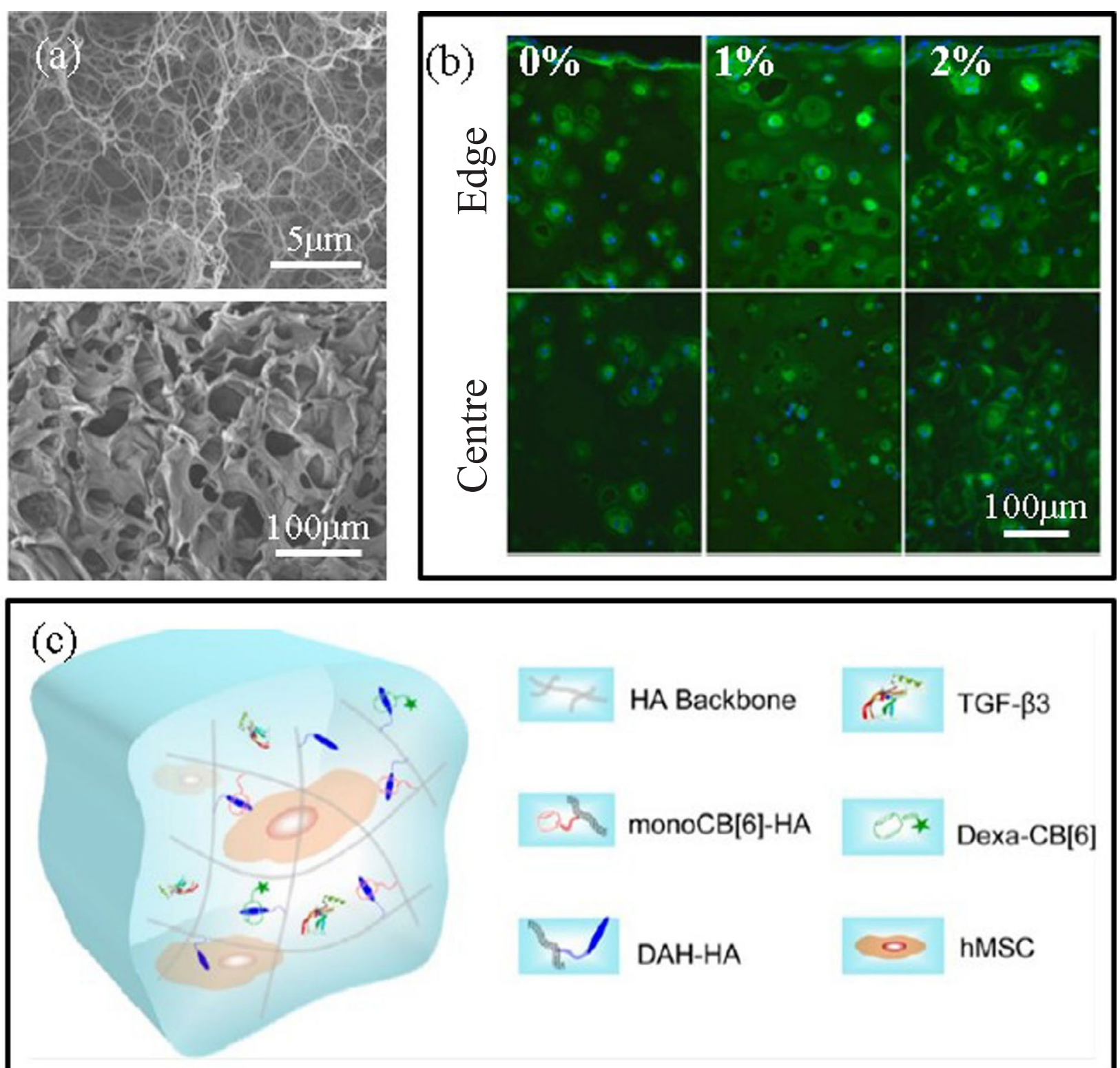

monoCB[6]-HA

Dexa-CB[6]

Fig. 2. (a) Scanning electron microscopy (SEM) of a fibrinogen hydrogel with (bottom) and without (top) interpenetrating methacrylated HA network (Snyder et al., 2014). (b) MSCs encapsulated in gelatin methacrylamide hydrogels exhibit more aggrecan (green) with increasing concentrations of methacrylated HA, which acts as a dual network (Levett et al., 2014). (c) Hydrogel molecular structures and crosslinking schemes can become quite complex, as seen by this schematic representation of supramolecular hydrogels prepared with CB[6]-HA, DAH-HA and drug conjugated Dexa-CB[6] (Jung et al., 2014).

exert post-transcriptional effects on collagen expression to influence construct composition as cells deposit matrix over time (Little et al., 2014).

\section{Double networks}

A double network consists of two networks with significantly different mechanical properties crosslinked together (Fig. 1d). Typically, the first network provides a rigid structure and the second network is ductile, resulting in greater toughness than the corresponding single networks alone would have achieved since the network can yield under mechanical load (Gong, 2010). These types of networks have gained interest in cartilage tissue engineering due to their superior mechanical properties over traditional hydrogels, including those that can approach the mechanics of native hyaline cartilage (Arnold et al., 2011). Double networks are also conceptually appealing since cartilage and other skeletal tissues inherently incorporate double networks into their ECM in order to achieve their robust mechanical properties (Arnold et al., 2011).

Commonly utilised double networks for cartilage tissue engineering consist of a combination of two acrylamide polymers, poly(2-acrylamido-2-methylpropanesulfonic acid) (PAMPs), poly(acrylamide) (PAAm) and/or poly(N,N-dimethylacrylamide) (PDMAAm) (Yasuda et al., 2009). Work with these double network hydrogels has recently extended to in vivo studies in both rabbit and sheep models of critical size defects, in which acellular plugs comprised of this double network implanted in defects resulted in enriched GAG and type II collagen content as 
measured by histological staining after 4 weeks (Kitamura et al., 2016). In all cases, the double-network plug was not infiltrated by cells and instead seemed to serve as a support material for tissue regeneration above and around it.

A direct comparison between double network hydrogels and traditional single network hydrogels of either only PAMPS or PDMAAm demonstrated that double network hydrogel constructs resulted in superior cartilage repair by histological scoring (Ogawa et al., 2012). The tissue formed in the presence of double networks also exhibited similar gene expression profiles (Imabuchi et al., 2011) and tissue surface roughness of native cartilage, even as observed by confocal laser scanning microscopy. Furthermore, combinatorial therapies using double network hydrogels and intra-articular injections (e.g. HA) have shown potential in tissue quality as measured by histological scoring and volume of cartilage generated by double network hydrogels implanted in cylindrical osteochondral defects in rabbit femoral trochlea at both 4 and 12 weeks, using acellular plugs of the PAMPS/ PDMAAm double network (Fukui et al., 2014).

However, the application of novel and cell-instructive materials for double network construct design in cartilage tissue engineering has not been widely explored until recently. Polymers used for double networks are often nondegradable, and while this stability renders them potentially useful materials for mechanical support in tissue defects, this stable and rigid network may limit cell infiltration and impede long-term matrix deposition and distribution. Despite these properties of double-network hydrogels, Levett and co-workers developed a double network consisting of a combination of gelatin-methacrylamide and hyaluronic acid-methacrylate. This system leveraged the relatively higher reactivity of methacrylate groups to create a double network, thus generating greater increases in compressive modulus and cartilage matrix component synthesis by encapsulated human chondrocytes (Levett et al., 2014). New hydrogels making use of this type of network are continuously being developed, such as an injectable and cytocompatible double network hydrogel based on HA that is formed through the combination of dynamic (i.e. guest-host pairs) and stable (i.e. covalent) crosslinks (Rodell et al., 2016).

\section{Dual networks}

Unlike double networks that use two materials with different mechanical properties, dual networks are defined as two materials crosslinked together into the same network and with similar crosslinking mechanisms (Fig. 1e). Although dual networks do not possess the toughness of double networks, each material in dual networks can imbue other useful properties to the hydrogel. For instance, one material can enable effective integration with the surrounding tissue, while the other can attract cells and encourage migration into the hydrogel. Moreira Teixeira et al. utilised a dextran-tyramine and heparin-tyramine dual network hydrogel to encapsulate bovine chondrocytes in vitro and reported improved cell viability and proliferation. Additionally, they observed increased deposition of chondroitin sulphate and collagen compared to cells in a single-component dextran-tyramine hydrogel (Moreira
Teixeira et al., 2012). Jin et al. (2011) reported similar findings using the same dual network polymers ex vivo with bovine articular cartilage explants $(0.5 \times 0.5 \times 0.2 \mathrm{~cm})$ to determine tissue-adhesion of the hydrogel constructs (Jin et al., 2011). In a more recent study, in situ forming ethylenediamino-functionalised HA (HA-EDA) and divinylsulfone-inulin (INU-DV) dual network hydrogels were used to encapsulate chondrocytes, with the first material selected due to its native presence in articular cartilage and the second for its biocompatibility and status as a widely-used FDA-approved polymer (Palumbo et al., 2015).

Along the same lines of using HA as the biologically relevant component, Pirinen and co-workers developed a dual network hydrogel using high molecular weight HA $(>1,600 \mathrm{kDa})$ and low molecular weight PVA $(27 \mathrm{kDa})$ functionalised with aldehydes and primary amines for crosslinking. This dual hydrogel system was amenable to tuneable swelling properties by varying the size of the smaller PVA component, and encapsulation of bovine knee chondrocytes showed favourable cell viability for at least 2 weeks in culture (Pirinen et al., 2015). Similar hydrogel systems (e.g. with HA and gelatin) have also produced favourable results in construct formation and maturation. Levett and colleagues reported that the addition of HA as the second component resulted in retention of a rounded chondrocyte morphology, greater aggrecan deposition and compressive moduli and suppression of type I collagen accumulation over the course of a $56 \mathrm{~d}$ study (Fig. 2b) (Levett et al., 2014).

\section{Guest-host networks}

Injectable hydrogels are an attractive approach for cartilage tissue engineering since they can be delivered via a direct injection or arthroscopically (Guvendiren et al., 2012). Shear-thinning hydrogels are a particularly important method towards this as a hydrogel can be disrupted during the injection process and then self-heal upon injection. One shear-thinning HA system developed is based on the reversible bonds between guest (adamantane modified HA) and host ( $\beta$-cyclodextrin modified HA) polymers, termed guest-host interactions (Fig. 1f) (Rodell et al., 2013). These types of interactions rapidly form hydrogels by noncovalent interactions in the guest-host bonds. In addition to cyclodextrin-adamantane guest-host pairs, guest-host interactions between cucurbit[6]uril and diaminohexane have also been investigated (Jung et al., 2014). Both of these interactions are reversible and give rise to shearthinning and self-healing hydrogels.

Wei and colleagues described the assembly of a two-component guest-host hydrogel with adamantanefunctionalised $\mathrm{HA}$ as the first component and monoacrylated $\beta$-cyclodextrin (assembled into clusters by photopolymerisation) as the second component (Wei et al., 2016). The hydrogels formed from these components exhibited nanoclustered interactions that allowed for robust drying and re-swelling without changes in water content or shape. They also enabled the chondrogenesis of encapsulated human MSCs with greater collagen deposition compared to a covalently crosslinked methacrylated HA network, although the differences in 


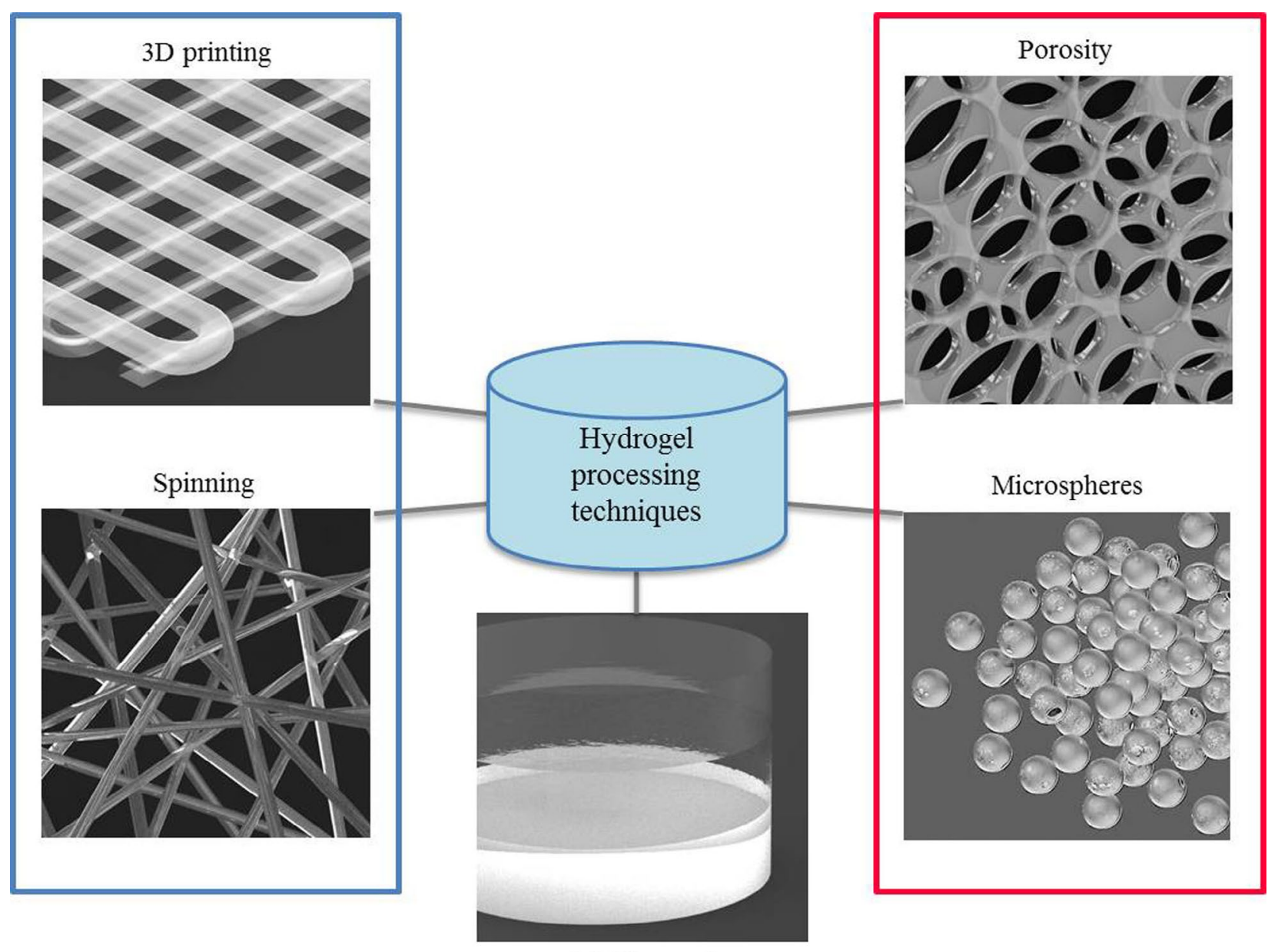

Multilayered

Fig. 3. Overview of different macroporous scaffold structures used for cartilage tissue engineering. To create hydrogel fibres, 3D printing and spinning techniques have been employed (blue box). In contrast, porous hydrogels and complementary microsphere hydrogels can also be fabricated (red box). To recapitulate native cartilage structures (e.g. different regions of cartilage, the osteochondral interface), multi-layer hydrogels incorporating several fabrication techniques can also be utilised (bottom).

biophysical properties and network structure limited a direct comparison (Wei et al., 2016). Jung et al. (2014) demonstrated the use of guest-host interactions between curcubit[6] uril and diaminohexane to create hydrogels for the encapsulation of human MSCs as well as simultaneous release of dexamethasone for chondrogenesis. Cells in this injectable guest-host system, especially with the additional function of controlled dexamethasone release, exhibited increased cell proliferation, GAG synthesis, chondrogenic gene expression (i.e. type II collagen, COMP, aggrecan, Sox9) and neocartilage formation in an in vivo subcutaneous study (Fig. 2c).

\section{Advances in the processing of hydrogel scaffolds}

Parallel advances in manufacturing technologies and material design have paved the way for new possibilities in how hydrogels can be manipulated and formed into 3D macroporous scaffolds. Architectures that aid viability, retention and chondrogenic induction of cells have been achieved using several techniques including 3D printing, spinning and doping hydrogels with degradable porogens or microspheres (Fig. 3). Due to their ability to generate unique microenvironments for cells and better recapitulate tissue structure on the macroscale, these techniques have become more prominent in tissue engineering in general and in cartilage engineering in particular. Recent studies making use of some of these more widely adopted methods are outlined in the subsections below.

\section{Hydrogel fibres}

Fibre fabrication techniques at length scales ranging from $150 \mathrm{~nm}$ to $1600 \mu \mathrm{m}$ are typically generated by either spinning or $3 \mathrm{D}$ printing. The resultant fibrous networks offer a number of potential advantages, from more facile cell infiltration and greater hydrogel surface area (and thus improved diffusion of nutrients and other soluble factors) to increased mechanical properties. The techniques involve both the encapsulation of cells within macrofibres to the seeding of meshes formed from nanofibres. Numerous groups have engineered hydrogel fibres using material composites and copolymers that allow for subsequent photo and ionic crosslinking, resulting in a wide spectrum of material properties. 


\section{$3 D$ printing}

$3 \mathrm{D}$ printing, or additive manufacturing, typically involves the building of 3D structures layer-by-layer with the controlled deposition of materials. A chief concern with $3 \mathrm{D}$ printing of cell-laden hydrogels is the optimisation of the printing parameters and control over material properties to enable efficient printing while preserving cell viability and phenotype. Schuurman et al. adapted a methacrylated gelatin formulation by introducing HA to increase the viscosity of the precursor material and demonstrated print fidelity and chondrocyte viability with post-print photocrosslinking (Schuurman et al., 2013). Conceptually similar fabrication techniques have been explored with other methods, including the use of ionically crosslinkable materials in lieu of photocrosslinking. For example, Fedorovich and colleagues used chondrocyte-laden alginate crosslinked with calcium chloride. In this case, constructs were printed with varying nozzle diameter, print speed and fibre orientation and then cultured or implanted subcutaneously to demonstrate cell viability and tissue formation (Fedorovich et al., 2012). Building upon this approach, Markstedt and co-workers engineered additional shear-thinning properties into this ionically crosslinkable network by incorporating nanofibrillar cellulose into the alginate precursors (Markstedt et al., 2015). Cui et al. (2012) examined the timing of crosslinking in this context as well, demonstrating direct $3 \mathrm{D}$ printing into osteochondral defects using a PEG dimethacrylate hydrogel in an explant model. The authors found greater chondrocyte viability and more uniform cell distribution with tandem (simultaneous) crosslinking, where crosslinking occurs at the same time as gel extrusion, rather than with traditional post-print crosslinking in which the construct can be crosslinked after printing (Cui et al., 2012).

Thermally responsive hydrogels use temperature to modulate their gelation behaviour, allowing for the transition from liquid to hydrogel, exclusively as a function of temperature. This is particularly appealing in cartilage tissue engineering, since the change from ambient temperature to physiological conditions can induce rapid gel formation (Klouda and Mikos, 2008) or stabilise materials in $3 \mathrm{D}$ printing processes. A common composite choice for thermoresponsive "bioinks" consists of HA or chondroitin sulphate mixed with a thermoresponsive polymer. Numerous groups have tested different thermoresponsive materials in this regard, from poly(N-isopropylacrylamide) (pNIPAAM) (Kesti et al., 2015) to triblock copolymers composed of PEG linked to $\mathrm{N}$-(2-hydroxypropyl) methacrylamide (HPMA) (Boere et al., 2015).

In exploring possible effects of thermoresponsive material structures at the nanoscale, Muller et al. showed that nanostructuring a thermoresponsive hydrogel allowed for high chondrocyte viability in a printed multilayer mesh and other more physiologically-relevant constructs including an ear (Fig. 4e) and a sheep meniscus (Fig. 4f,g) (Muller et al., 2015). In this case, the authors incorporated both unmodified and acrylated Pluronic, crosslinked and then subsequently washed out the unmodified fraction. In each case mentioned, methacrylated HA or chondroitin sulphate was either used as a crosslinker or as an independent reinforcing network to compensate for the generally poor mechanics of the thermoresponsive component.

\section{Spinning hydrogel fibres}

A number of techniques that use spinning technology have been explored towards fabricating hydrogel fibres. A wetspinning technique was introduced by Han and colleagues in which an 8-arm methacrylated PEG precursor was injected through a syringe pump into a tris(2-aminoethyl) amine (TAEA) bath (Han et al., 2014). Subsequent crosslinking yielded microribbons that could be further crosslinked together to form hydrogels with complex geometries at both the micro and macro scales, and these hydrogels supported adipose-derived stem cell viability as well as proliferation and spreading under various conditions with the potential of recapitulating the laminar matrix organisation of cartilage tissue (Han et al., 2014).

Electrospinning has also been used to generate fibrous constructs from methacrylated HA that supported human MSC interactions and chondrogenesis, without outcomes dependent on the hydrogel fibre properties such as mechanics and adhesion (Kim et al., 2013). Multi-polymer fibrous scaffolds were fabricated using HA hydrogels and implanted in combination with microfracture for tissue repair in a mini-pig model. The multi-polymer design allowed for stable fibres to maintain the fibre structure and degradable fibres to release chondroinductive factors. Here, the transforming growth factor beta 3 (TGF- $\beta 3$ ) spun into degradable HA fibres was verified to be active upon release and in vivo it resulted in improved histological scores and type II collagen content in the repaired defects (Kim et al., 2015). Fibre spinning and printing have also been combined in cell-laden constructs, where, for instance, alternating rounds of electrospinning of polycaprolactone (PCL) fibres and printing of fibrin-collagen hydrogels were performed to yield scaffolds with greater mechanical properties and improved histological staining of GAGs and type II collagen in an 8 week subcutaneous implant study in mice (Xu et al., 2013).

\section{Porous hydrogels}

While hydrogel fibres and scaffolds comprised of fibrous networks permit the incorporation of pores of varying shapes and sizes, several other methods for generating porous hydrogel constructs within the context of cartilage tissue engineering also exist. These may be advantageous in some cases, since they do not need specialised printing or spinning equipment and may produce scaffolds more rapidly. More recently, the design of porous hydrogels has turned to dynamic control of macroporosity as well as dynamic control of bulk material properties.

\section{Porogen approaches}

Han et al. developed stimuli-responsive porogens from alginate, gelatin and HA that respond to chelation, temperature and enzymatic activity. The alginate porogen was also used to deliver chondrocytes into the hydrogel and treatment with changes in temperature, EDTA or hyaluronidase activity released chondrocytes from the alginate components and subsequently increased hydrogel 

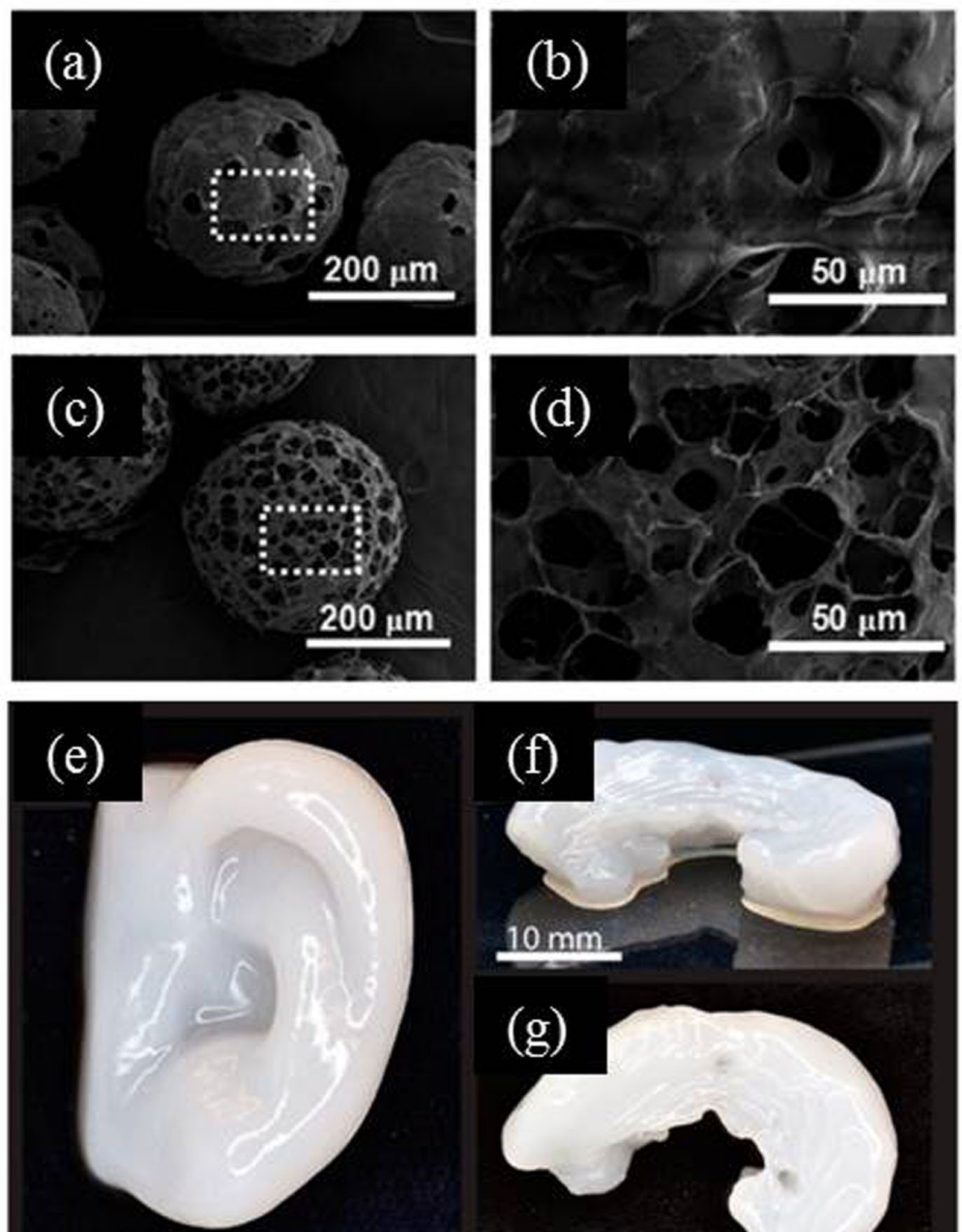

\section{$10 \mathrm{~mm}$}
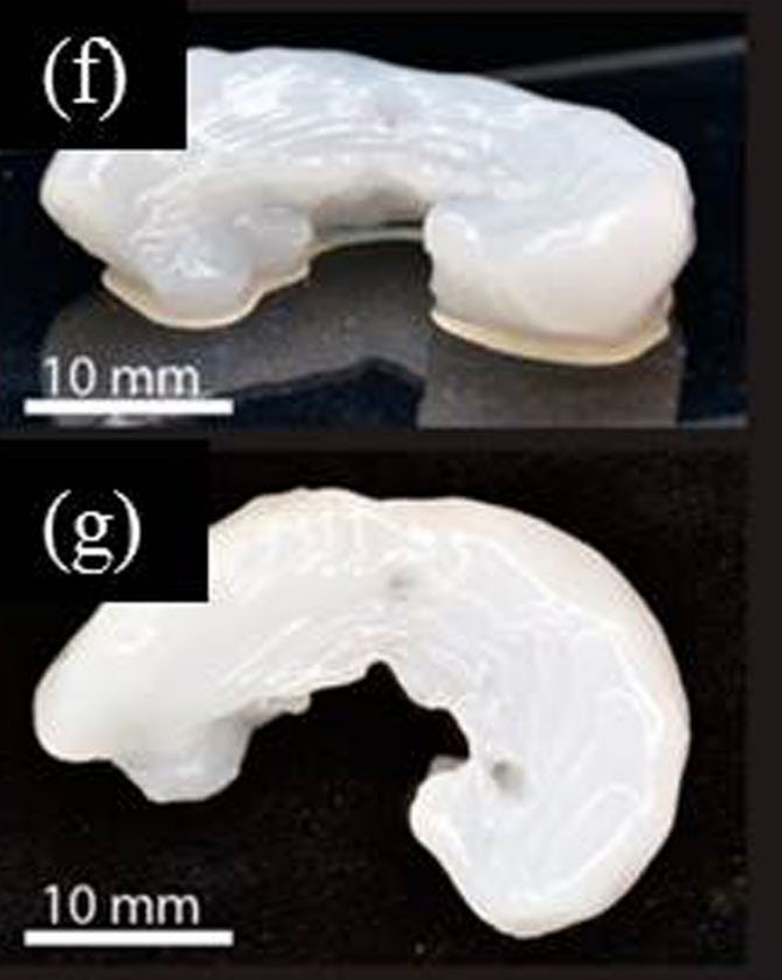

Fig. 4. SEM of PBLG microsphere hydrogels fabricated at a gelatin concentration of (a,b) $1.9 \%$ and $(\mathbf{c}, \mathbf{d}) 3.25 \%$ (Fang et al., 2015). 3D printing was used to print (e) a human ear and (f,g) a sheep meniscus, as seen from different angles with Ink8020 after crosslinking (Muller et al., 2015). 
macroporosity (Han et al., 2013). While this controlled degree of macroporosity affected bulk mechanical properties, approaches have also been developed where mechanics can be dynamically increased with photocrosslinking, such as from 2.6 to $12.5 \mathrm{kPa}$, without changing porosity (Marklein et al., 2012). In this case, across the range of mechanics tested, chondrogenic gene expression by human MSCs in porous constructs was slightly upregulated even in the absence of chondrogenic media (Marklein et al., 2012). In a different approach, Ahrem et al. (2014) treated whole bacterial nanocellulose hydrogels with $3 \mathrm{D}$ laser perforation to produce a porous construct that promoted chondrocyte ingrowth and proliferation. Control of hydrogel porosity and functionalisation of pore interiors with relevant molecules such as type II collagen have also been explored in tandem with the use of stimuli-responsive materials. For example, Almeida and colleagues recently showed that "shape-memory" properties can be attained by covalent crosslinking of alginate scaffolds by making use of carbodiimide chemistry, which along with type II collagen incorporation led to higher sulphated GAG (sGAG) and collagen production in comparison to scaffolds functionalised with type I collagen (Almeida et al., 2016).

\section{Microsphere hydrogels}

As a complement or alternative to porous bulk hydrogels, scaffolds comprised of microspheres from a range of materials have also been developed. Such scaffolds have been studied most often as delivery vehicles or depots for small molecules or proteins, but they have also been used as potential cell carriers (i.e. cell microencapsulations). In one case, a xanthan gum derivative was selected as the encapsulation material because of its established safety record in pharmaceuticals, food and cosmetics, as well as its observed protective effects on joint cartilage administered via an intra-articular injection (Hamcerencu et al., 2007; Katzbauer, 1998). Murine chondrocytes were viable in these microgels for up to $21 \mathrm{~d}$ (Mendes et al., 2012). Another interesting scaffold, comprised of the synthetic peptide poly(g-benzyl-L-glutamate) (PBLG), achieved tuneable porosity in microspheres by varying the amount of gelatin porogen followed by its removal, producing spherical hydrogel scaffolds that could be seeded with chondrocytes throughout their interior (Fig. 4a-d). In vitro, they retained chondrocyte roundness after seeding and supported ECM deposition in culture for 3 to $7 \mathrm{~d}$ and, when injected subcutaneously in vivo, they formed tissue with improved histological scoring and type II collagen content compared to cell-only injections (Fang et al., 2015).

\section{Multilayered scaffolds}

The final scale of architectural complexity in cartilage engineering is one that attempts to recapitulate the zonal architecture of cartilage in the joint. Regardless of the particular technique used to deposit or arrange the material, numerous groups have worked to establish two-to-three-layered hydrogel constructs with differential characteristics to mimic the zones likely damaged by osteochondral defects. Feasibility of a 3D printed bilayer hydrogel construct has been demonstrated, both by 3D extrusion of material (Cui et al., 2012) and by projection stereolithography (Sun et al., 2015). As for tri-layer hydrogels, Nguyen and co-workers described a PEG-based hydrogel with the superficial layer containing chondroitin sulphate and a matrix metalloproteinase (MMP)-sensitive peptide, the middle layer containing PEG and chondroitin sulphate and the deep layer composed of PEG and HA. In such a construct the investigators showed that with a single stem cell population evenly distributed in all layers, type II collagen deposition decreased gradually from superficial to deep layers along with an increase in type $\mathrm{X}$ collagen and proteoglycans, resulting in a gradient of compressive modulus across the construct (Nguyen et al., 2011).

However, there are a number of different approaches to zonal design of hydrogel constructs, with alternatives including the use of different cell types (e.g. chondrocytes, osteogenic progenitors) (Fedorovich et al., 2012) or culture conditions in each layer rather than substantially modifying the material characteristics of the layer itself. It remains to be seen how advances in the design of hydrogels with this degree of control over architecture will compare with other diverse strategies for hydrogel engineering towards cartilage repair.

\section{Controlled presentation and delivery of biochemical factors}

Cartilage formation is regulated by the dynamic spatial and temporal presentation of an assortment of biochemical factors that either interact with receptors at the cell surface or are internalised by cells, resulting in the induction of chondrogenesis. Thus, hydrogels are being designed where biomolecules are either directly tethered to the hydrogel or are encapsulated within the hydrogel for presentation to entrapped or surrounding cells. This control over biomolecules' spatial introduction and timing can aid in the production of heterogeneous constructs to mimic developing or adult tissues and to enhance the dynamic processes of differentiation.

The spatial and temporal presentation of biochemical factors is extremely important, since uncontrolled exposure may not only reduce effects on chondrogenesis and cartilage formation, but can also induce adverse effects. For example, supra-physiological levels of TGF- $\beta$ delivered via injections to murine knee joints resulted in a decrease in proteoglycan synthesis, synovial fibrosis and endochondral ossification (Bakker et al., 2001; van Beuningen et al., 2000). To regulate the dose and timing of biochemical factors that interact with cell surface receptors, several techniques such as protease-degradable tethers and presenting factors in their latent form (Place et al., 2012; Re'em et al., 2012) have been recently proposed. To achieve controlled release of cartilage-inducing factors that are internalised by cells, novel design techniques towards the improved design of carrier vehicles [e.g. microparticles (MPs) or nanoparticles (NPs)] loaded with biochemical factors have been proposed. In this section we will highlight several recent advances in biochemical factor presentation using these mechanisms and their in vivo and in vitro cartilage regeneration potential using cell-laden and acellular hydrogels (Fig. 5). 


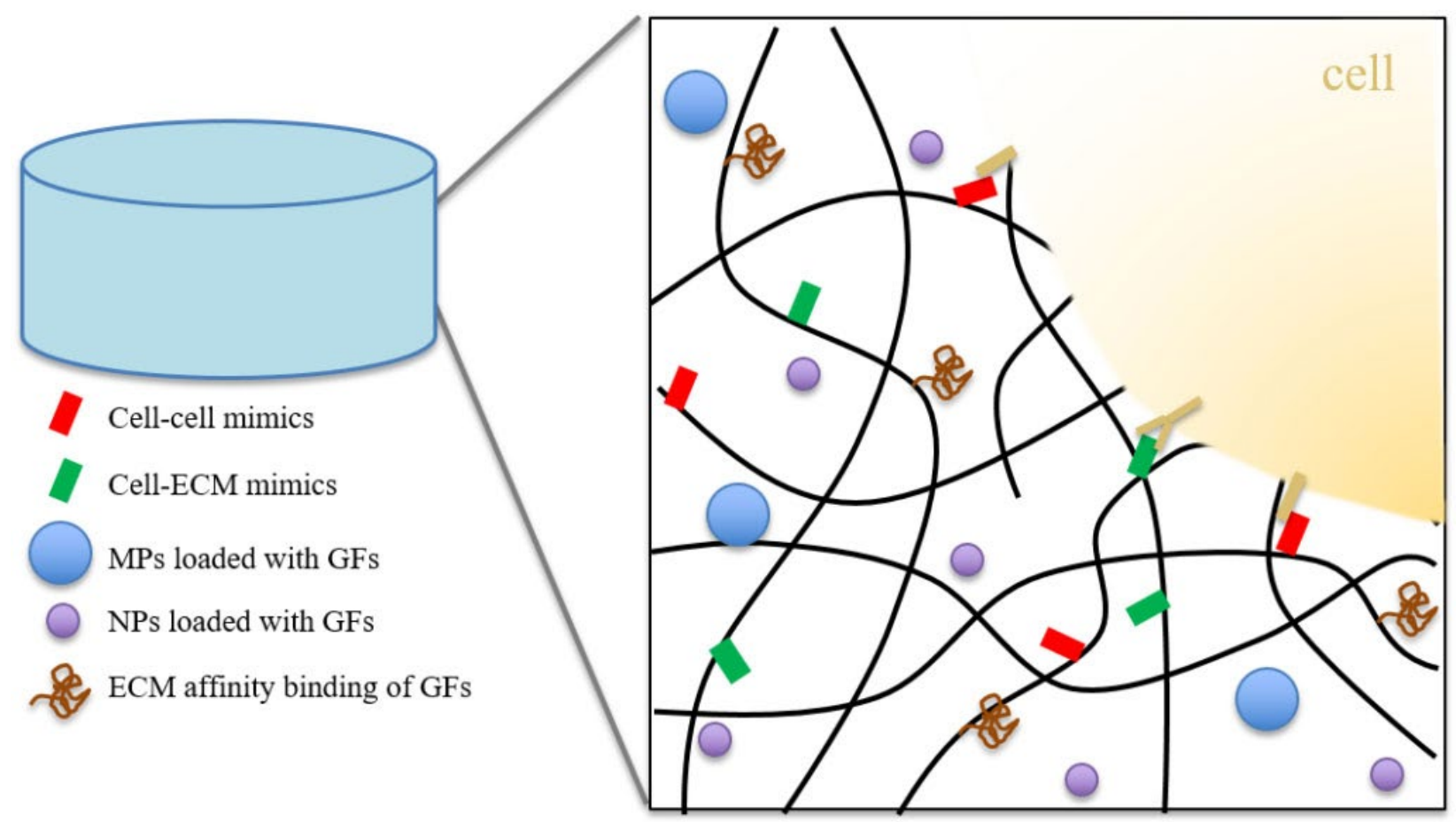

Fig. 5. Overview of controlled presentation of biochemical factors. These include cell-cell and cell-matrix interactions, as well as growth factors and other molecules that can be either tethered to the hydrogels by affinity or heparin binding, or encapsulated in MPs and NPs for controlled release.

\section{Covalent conjugation and affinity binding}

In order to present cells with biochemical cues that interact with integrins or receptors at the cell surface, these signals are typically incorporated into the hydrogel itself, either by covalent tethering or affinity binding by electrostatic interactions as is observed within the ECM. Chemical coupling of peptides to hydrogels is one of the simplest approaches that can be taken to modify hydrogels with biochemical signals.

During limb development, the first instance of cartilage formation occurs within a highly condensed cell-rich aggregate that is largely devoid of ECM (Bobick et al., 2009). Cell-cell interactions in this context are mediated by the adhesion molecule N-cadherin (DeLise and Tuan, 2002a; DeLise and Tuan, 2002b; Oberlender and Tuan, 1994) and these interactions regulate signalling events that are critical to the initiation of chondrogenesis. Of note, these cell-cell adhesion signals are not present throughout development, but rather arise and peak during this condensation phase, after which adhesion-based signalling from the formed ECM dominates (Singh and Schwarzbauer, 2012). To this end, Bian et al. recently developed an HA hydrogel system that incorporates an $\mathrm{N}$-cadherin mimetic peptide (i.e. HAV) (Fig. 6a) and found that the peptide conjugation promoted both early chondrogenesis of human MSCs, as well as cartilagespecific matrix production (Fig. 6b) (Bian et al., 2013). This outcome was even observed in vivo, and it was shown recently that the presence of the $\mathrm{N}$-cadherin signal from the hydrogel also influenced $\beta$-catenin signalling in human MSCs (Fig. 6c,d) (Vega et al., 2016).

Due to the importance of the temporal presentation of biochemical signals, there has been increased interest in regulating the presentation of these biochemical cues by enzymatic regulation. Towards this, Salinas and Anseth used thiol-acrylate photopolymerisation to tether RGD peptides with an MMP-13 cleavable linker in order to induce MSCs towards a chondrogenic phenotype. The authors found that MSCs encapsulated in these hydrogels produced active MMP-13 between 9 and $14 \mathrm{~d}$ in culture, resulting in an over ten-fold increase of GAG deposition in comparison to MSCs exposed to non-cleavable RGD peptides (Salinas and Anseth, 2008).

Besides peptides, growth factors, such as those from the TGF- $\beta$ superfamily, have also been tethered to hydrogels. Although TGF- $\beta$ plays a key role in promoting chondrogenesis, its therapeutic utility is limited by its inherent protein instability, requiring high amounts of protein that can cause adverse side effects with inefficient cartilage formation. Choi and co-workers compared hydrogels containing TGF- $\beta 1$ covalently tethered to visible blue light-inducible chitosan (MeGC) hydrogels prior to photopolymerisation with type II collagen versus MeGC hydrogels with positively-charged TGF- $\beta 1$ ionically conjugated to type II collagen. The authors found that the cumulative release of TGF- $\beta 1$ was significantly higher in the covalently bound system, resulting in more sGAG production by adipose-derived stem cells in vitro, as well as improved integration with surrounding tissue of hydrogels implanted in a rat cartilage defect model (Choi et al., 2015).

In vivo, control over the bioavailability of biomolecules is dependent on numerous factors. For example, growth factors including TGF- $\beta$ are present as a complex including a latency associated peptide (LAP), which inactivates TGF- $\beta$ by masking the receptor-binding domains (Shi et al., 2011). As such, members of the TGF- $\beta$ superfamily 

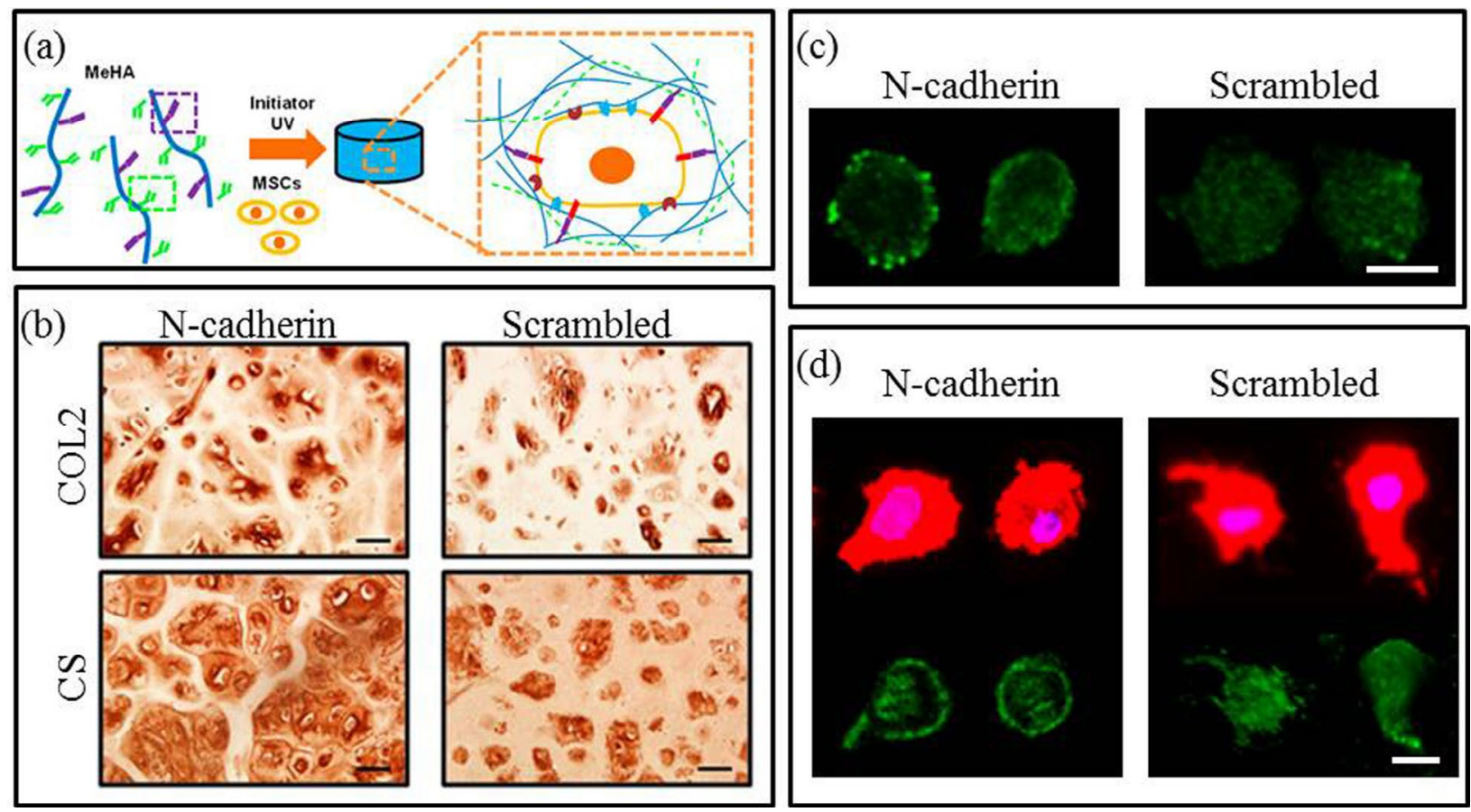

Fig. 6. Human MSCs were (a) photoencapsulated in hydrogels containing either N-cadherin mimics or scrambled sequence controls. (b) After 4 weeks of in vitro culture, N-cadherin mimics enhanced chondroitin sulphate (CS) and type II collagen (COL2) production by human MSCs, as seen by immunohistochemical staining (Bian et al., 2013). (c,d) Single cell analysis of MSCs in these hydrogel environments showed an increase in N-cadherin mediated $\beta$-catenin signalling after $3 \mathrm{~d}$ in culture. (c) Cross-sectional images of MSCs stained for $\beta$-catenin (green) show that $\mathrm{N}$-cadherin mimics recruit $\beta$-catenin to the cell membrane. Additionally, $\mathrm{N}$-cadherin mimics induced an increase in nuclear $\beta$-catenin, as confirmed by (d) representative maximum (top) and average (bottom) projections of single MSCs stained for actin (red), nucleus (blue) and $\beta$-catenin (green) (Vega et al., 2016). Scale bars: $\mathbf{b}=50 \mu \mathrm{m} ; \mathbf{c}, \mathbf{d}=5 \mu \mathrm{m}$.

remain inactive and protected from degradation until LAP is removed by one of several mechanisms including proteolytic cleavage of LAP. Place et al. sought to recapitulate this natural mechanism by coupling TGF- $\beta 1$ in its latent form to PEGDA prior to crosslinking with thiolated HA hydrogels by a Michael addition reaction (Place et al., 2012). The authors found that encapsulated chondrocytes in these hydrogels were viable for up to $34 \mathrm{~d}$ and produced type II collagen without the presence of soluble TGF- $\beta 1$, as confirmed by immunocytochemistry (Place et al., 2012).

In addition to covalent conjugation, affinity binding of macromolecules to growth factors is an alternative method towards the controlled release of growth factors. To this end, dendrimers, a particular type of branched macromolecules, can be functionalised with peptides that selectively bind to different molecules (Simon-Gracia et al., 2013). Seelbach and colleagues recently investigated the use of dendrimers with affinity binding peptides towards the non-burst release of bone morphogenetic protein 2 (BMP-2) and TGF- $\beta 1$. Briefly, dendrimers containing four binding peptides presenting sequences targeting BMP-2 or TGF- $\beta 1$ protein binding were covalently conjugated to HA, mixed with poly(N-isopropylacrylamide)-grafted HA (Hyal-pN) brush copolymers, and then loaded with BMP-2 or TGF- $\beta 1$. The authors found a dependence of the release of these growth factors based on the length of the dendrimer arms (Seelbach et al., 2015).
GAGs are highly negatively-charged polysaccharides that can modulate macromolecular binding, particularly with positively-charged molecules (Lindahl and Hook, 1978). By regulating the degree of GAG sulphation, the extent of this charge can be tuned to control the presentation of positively-charged growth factors (e.g. TGF- $\beta 1)$. To this extent, Lim and Temenoff showed that MSCs cultured in TGF- $\beta 1$ containing media and encapsulated in desulphated chondroitin hydrogels featured significantly upregulated gene expression of type II collagen and aggrecan when compared to a PEG-based control hydrogel (Lim and Temenoff, 2013).

\section{Particles for controlled release}

Carrier vehicles such as MPs and NPs as a method to deliver encapsulated factors have been extensively studied and optimised to achieve continuous delivery of their payload by controlling the chemical (e.g. charge, degradability) and physical (e.g. carrier size, shell thickness) properties of the carriers. In contrast to simple diffusion of soluble cues into hydrogel constructs, loaded MPs encapsulated in hydrogels protect biochemical factors from degradation and help prevent rapid diffusion and clearance from hydrogels, allowing for greater control over the release kinetics of biochemical cues into the hydrogel. Although this technique has been used and investigated for decades, the utility of MPs (Reyes et al., 2013a; Reyes et al., 2013b) and NPs (Ertan et al., 2013; Lim et al., 2011; Siu et al., 
Table 1. Summary of hydrogel fabrication techniques for cartilage tissue engineering.

\begin{tabular}{|c|c|c|c|}
\hline \multicolumn{2}{|c|}{ Improvements in hydrogel structure } & \multicolumn{2}{|c|}{ Advances in the processing of hydrogel scaffolds } \\
\hline IPNs & $\begin{array}{l}\text { (Dinescu et al., 2015; Guo et al., } \\
\text { 2012; Ingavle et al., 2013; Snyder } \\
\text { et al., 2014) }\end{array}$ & $3 \mathrm{D}$ printing & $\begin{array}{l}\text { (Fedorovich } \text { et al., 2012; Kesti } \text { et al., 2015; } \\
\text { Markstedt } \text { et al., 2015; Muller } \text { et al., 2015; } \\
\text { Schuurman } \text { et al., 2013) }\end{array}$ \\
\hline Semi-IPNs & $\begin{array}{l}\text { (Little et al., 2014; Park et al., } \\
\text { 2013; Skaalure } \text { et al., 2014) }\end{array}$ & Spinning & (Han et al., 2014; Xu et al., 2013) \\
\hline Double networks & $\begin{array}{l}\text { (Arnold et al., 2011; Kitamura et } \\
\text { al., 2016) }\end{array}$ & Porogens & $\begin{array}{l}\text { (Ahrem et al., 2014; Han et al., 2013; Marklein } \\
\text { et al., 2012) }\end{array}$ \\
\hline Dual networks & $\begin{array}{l}\text { (Jin et al., 2011; Levett et al., } \\
\text { 2014; Moreira Teixeira et al., 2012; } \\
\text { Palumbo et al., 2015; Pirinen et al., } \\
\text { 2015) }\end{array}$ & Microspheres & (Fang et al., 2015; Mendes et al., 2012) \\
\hline $\begin{array}{l}\text { Guest-host } \\
\text { networks }\end{array}$ & (Jung et al., 2014; Wei et al., 2016) & Multilayered & $\begin{array}{l}\text { (Cui et al., 2012; Fedorovich et al., 2012; } \\
\text { Nguyen et al., 2011; Sun et al., 2015) }\end{array}$ \\
\hline \multicolumn{4}{|c|}{ Controlled presentation and delivery of biochemical factors } \\
\hline Mimetic peptides & \multicolumn{3}{|c|}{ (Bian et al., 2013; Salinas and Anseth, 2008; Vega et al., 2016) } \\
\hline $\mathrm{MPs} / \mathrm{NPs}$ & \multicolumn{3}{|c|}{ (Ahearne and Kelly, 2013; Siu et al., 2012; Spiller et al., 2012) } \\
\hline $\begin{array}{l}\text { ECM affinity } \\
\text { binding }\end{array}$ & \multicolumn{3}{|l|}{ (Choi et al., 2015; Seelbach et al., 2015) } \\
\hline
\end{tabular}

2012; Whitmire et al., 2012) has seen recent advances in their use as delivery vehicles for cartilage repair.

To achieve a more sustained delivery of growth factors, Spiller and co-workers recently designed a hybrid scaffold where insulin-like growth factor 1 (IGF-1) was loaded into a poly(vinyl alcohol) (PVA) and poly(lactic-co-glycolic acid) (PLGA) hydrogel system. The authors used a novel double emulsion technique, such that PLGA MPs $(11.3 \pm 6.4 \mu \mathrm{m})$ containing IGF-1 directly formed and evenly dispersed throughout the PVA hydrogel. IGF-1 release was linear and sustained for at least $45 \mathrm{~d}$, and in vivo studies showed that the hydrogel resulted in the formation of thick cartilage layers and exhibited good integration between the formed cartilage and the surrounding neocartilage (Spiller et al., 2012).

In addition to IGF-1, there are other biochemical cues that enhance cartilage formation and can benefit from a suitable delivery vehicle for their sustained release. Nell-like molecule 1 (Nell-1) has previously been shown to induce differentiation and growth towards bone and cartilage tissue in vivo (Siu et al., 2011). In order to showcase the importance of sustained release of Nell-1, Siu et al. compared the release of Nell-1 from alginate hydrogels either directly encapsulated in the bulk hydrogel, or loaded into chitosan NPs (100-300 nm in size). The authors found that Nell-1 containing NPs induced a significantly more gradual release of the protein in comparison to hydrogels where Nell-1 was directly encapsulated. Additionally, Nell$1 \mathrm{NP}$ containing hydrogels induced an increase in GAG synthesis and proteoglycan accumulation in a critical size osteochondral defect 12 weeks post-implantation (Siu et al., 2012). This study showed not only the importance of Nell-1 in cartilage formation, but also the need for NPs for controlled release.

Although design parameters used to control the release kinetics of biochemical factors loaded in MPs and NPs have been thoroughly investigated, not much work has been done to study the influence of the encapsulating hydrogel on the release profiles of biochemical cues loaded in MPs and NPs. To this end, Ahearne and co-workers studied how different hydrogel macromers (i.e. fibrin, agarose and gellan gum) affected the efficiency of TGF- $\beta 3$ loaded MPs to induce chondrogenesis of encapsulated MSCs. Piglet MSCs and TGF- $\beta 3$ loaded gelatin MPs (50$70 \mu \mathrm{m})$ were encapsulated in fibrin, agarose or gellan gum hydrogels, and the role of the MPs in inducing cartilage formation from the MSCs over the course of $21 \mathrm{~d}$ in vitro was evaluated. The authors observed a significant increase in sGAG accumulation in the agarose and gellan gum hydrogels in comparison to the fibrin hydrogels. Additionally, histological staining with alcian blue showed a stronger and more homogeneous distribution of SGAG in the gellan gum hydrogels, in contrast to a more pericellular presentation of sGAG in the agarose hydrogels (Ahearne and Kelly, 2013). This study shows that not just MPs and NPs, but the hydrogel macromers as well, are important determinants of the release profiles of biochemical factors loaded in these vehicle carriers.

\section{Summary and future directions}

Although hyaline cartilage is perceived as a simple tissue, developing biomaterials that can achieve mechanical properties of native cartilage and complete integration with surrounding tissues remains a challenge. Cartilage tissue engineering has seen a rapid advance in fabrication techniques, resulting in hydrogel constructs that are improving the quality of produced cartilage (summarised in Table 1). To increase the mechanical properties of hydrogels, traditional single network hydrogels are being supplemented with either additional networks or mixtures of polymers. These techniques have also been used to fabricate hydrogels which can induce integration with surrounding tissue while promoting chondrogenesis in vivo. Novel processing techniques have also been employed towards fabricating hydrogel fibres and porous hydrogels for improved cartilage formation, as well as 
the fabrication of multilayered hydrogels that mimic the zonal architecture of native cartilage. In addition to these advances, the field has also seen an increased interest in presenting biochemical cues in a controllable and temporal fashion.

Looking ahead, combinatorial screening approaches to robustly identify synergies between the various hydrogel parameters presented in this review would enable the development of cell-laden and acellular hydrogels that will eventually adequately recapitulate native cartilage. The technology to achieve this kind of screening has been explored for the discovery of hydrogels with a variety of properties, such as cell adhesion, proliferation and foreign body responses (Le et al., 2016; Vegas et al., 2016; Zant and Grijpma, 2016a; Zant and Grijpma, 2016b). So far this has been achieved by changing the extent of material modification, the combinations of different materials at different ratios or the amount of a peptide of interest in the precursor. Although none of these combinatorial hydrogel platforms have been directly applied to probe chondrogenesis or cartilage matrix synthesis, they are extremely promising for this application. Additionally, a better understanding of cartilage both during development and in its mature state will be critical to the success of advancing these exciting techniques towards developing hydrogel systems that robustly recapitulate native cartilage.

\section{Acknowledgement}

The authors would like to acknowledge funding from the National Institutes of Health (T32 AR007132, R01 EB008722) and the National Science Foundation for a graduate research fellowship (to MK).

We wish to confirm that there are no known conflicts of interest associated with this publication and there has been no significant financial support for this work that could have influenced its outcome.

\section{References}

Ahearne M, Kelly DJ (2013) A comparison of fibrin, agarose and gellan gum hydrogels as carriers of stem cells and growth factor delivery microspheres for cartilage regeneration. Biomed Mater 8: 035004.

Ahmed TA, Hincke MT (2010) Strategies for articular cartilage lesion repair and functional restoration. Tissue Eng Part B Rev 16: 305-329.

Ahrem H, Pretzel D, Endres M, Conrad D, Courseau J, Muller H, Jaeger R, Kaps C, Klemm DO, Kinne RW (2014) Laser-structured bacterial nanocellulose hydrogels support ingrowth and differentiation of chondrocytes and show potential as cartilage implants. Acta Biomater 10: 1341-1353.

Almeida H, Sathy BN, Dudurych I, Buckley CT, O’Brien FJ, Kelly DJ (2016) Anisotropic shape-memory alginate scaffolds functionalized with either type I or type II collagen for cartilage tissue engineering. Tissue Eng Part A. 23: 55-68.
Arnold MP, Daniels AU, Ronken S, Garcia HA, Friederich NF, Kurokawa T, Gong JP, Wirz D (2011) Acrylamide polymer double-network hydrogels: candidate cartilage repair materials with cartilage-like dynamic stiffness and attractive surgery-related attachment mechanics. Cartilage 2: 374-383.

Bakker AC, van de Loo FA, van Beuningen HM, Sime P, van Lent PL, van der Kraan PM, Richards CD, van den Berg WB (2001) Overexpression of active TGF-beta-1 in the murine knee joint: evidence for synovial-layerdependent chondro-osteophyte formation. Osteoarthritis 9: $128-136$.

Balakrishnan B, Banerjee R (2011) Biopolymer-based hydrogels for cartilage tissue engineering. Chem Rev 111: 4453-4474.

Benthien JP, Behrens P (2011) The treatment of chondral and osteochondral defects of the knee with autologous matrix-induced chondrogenesis (AMIC): method description and recent developments. Knee Surg Sports Traumatol Arthrosc 19: 1316-1319.

Bian L, Guvendiren M, Mauck RL, Burdick JA (2013) Hydrogels that mimic developmentally relevant matrix and $\mathrm{N}$-cadherin interactions enhance MSC chondrogenesis. Proc NatL Acad Scie U S A 110: 10117-10122.

Bobick BE, Chen FH, Le AM, Tuan RS (2009) Regulation of the chondrogenic phenotype in culture. Birth Defects Res C Embryo Today 87: 351-371.

Boere KWM, Blokzijl MM, Visser J, Linssen JEA, Malda J, Hennink WE, Vermonden T (2015) Biofabrication of reinforced 3D-scaffolds using two-component hydrogels. J Mater Chem B 3: 9067-9078.

Brittberg M (2008) Autologous chondrocyte implantation-technique and long-term follow-up. Injury 39 Suppl 1: S40-49.

Choi B, Kim S, Fan JB, Kowalski T, Petrigliano F, Evseenko D, Lee M (2015) Covalently conjugated transforming growth factor-beta 1 in modular chitosan hydrogels for the effective treatment of articular cartilage defects. Biomater Sci 3: 742-752.

Chung C, Mesa J, Randolph MA, Yaremchuk M, Burdick JA (2006) Influence of gel properties on neocartilage formation by auricular chondrocytes photoencapsulated in hyaluronic acid networks. J Biomed Mater Res A 77: 518-525.

Cui XF, Breitenkamp K, Finn MG, Lotz M, D’Lima DD (2012) Direct human cartilage repair using threedimensional dioprinting technology. Tissue Eng Part A 18: 1304-1312.

DeLise AM, Tuan RS (2002a) Alterations in the spatiotemporal expression pattern and function of $\mathrm{N}$-cadherin inhibit cellular condensation and chondrogenesis of limb mesenchymal cells in vitro. J Cell Biochem 87: 342 359.

DeLise AM, Tuan RS (2002b) Analysis of N-cadherin function in limb mesenchymal chondrogenesis in vitro. Dev Dyn 225: 195-204.

Dinescu S, Galateanu B, Radu E, Hermenean A, Lungu A, Stancu IC, Jianu D, Tumbar T, Costache M (2015) A $3 \mathrm{D}$ porous gelatin-alginate- based-IPN acts as an efficient promoter of chondrogenesis from human adipose-derived stem cells. Stem Cells Int DOI: 10.1155/2015/252909. 
Ertan AB, Yilgor P, Bayyurt B, Calikoglu AC, Kaspar C, Kok FN, Kose GT, Hasirci V (2013) Effect of double growth factor release on cartilage tissue engineering. $\mathrm{J}$ Tissue Eng Regen Med 7: 149-160.

Fang JJ, Yong Q, Zhang KX, Sun WT, Yan SF, Cui L, Yin JB (2015) Novel injectable porous poly(gammabenzyl-L-glutamate) microspheres for cartilage tissue engineering: preparation and evaluation. J Mater Chem B 3: 1020-1031.

Fedorovich NE, Schuurman W, Wijnberg HM, Prins HJ, van Weeren PR, Malda J, Alblas J, Dhert WJA (2012) Biofabrication of osteochondral tissue equivalents by printing topologically defined, cell-laden hydrogel scaffolds. Tissue Eng Part C Methods 18: 33-44.

Fukui T, Kitamura N, Kurokawa T, Yokota M, Kondo E, Gong JP, Yasuda K (2014) Intra-articular administration of hyaluronic acid increases the volume of the hyaline cartilage regenerated in a large osteochondral defect by implantation of a double-network gel. J Mater Sci Mater Med 25: 1173-1182.

Goldring MB (2006) Update on the biology of the chondrocyte and new approaches to treating cartilage diseases. Best Pract Res Clin Rheumatol 20: 1003-1025.

Gong JP (2010) Why are double network hydrogels so tough? Soft Matter 6: 2583-2590.

Guo Y, Yuan T, Xiao ZW, Tang PP, Xiao YM, Fan YJ, Zhang XD (2012) Hydrogels of collagen/chondroitin sulfate/hyaluronan interpenetrating polymer network for cartilage tissue engineering. J Mater Sci Mater Med 23: 2267-2279.

Guvendiren M, Lu HD, Burdick JA (2012) Shearthinning hydrogels for biomedical applications. Soft Matter 8: $260-272$.

Hamcerencu M, Desbrieres J, Popa M, Khoukh A, Riess G (2007) New unsaturated derivatives of xanthan gum: synthesis and characterisation. Polymer 48: 1921-1929.

Han LH, Lai JH, Yu S, Yang F (2013) Dynamic tissue engineering scaffolds with stimuli-responsive macroporosity formation. Biomaterials 34: 4251-4258.

Han LH, Tong XM, Yang F (2014) Photo-crosslinkable PEG-based microribbons for forming 3D macroporous scaffolds with decoupled niche properties. Adv Mater 26: 1757-1762.

Hunziker EB (2002) Articular cartilage repair: basic science and clinical progress. A review of the current status and prospects. Osteoarthritis Cartilage 10: 432-463.

Imabuchi R, Ohmiya Y, Kwon HJ, Onodera S, Kitamura N, Kurokawa T, Gong JP, Yasuda K (2011) Gene expression profile of the cartilage tissue spontaneously regenerated in vivo by using a novel double-network gel: comparisons with the normal articular cartilage. BMC Musculoskelet Disord 12: 213.

Ingavle GC, Frei AW, Gehrke SH, Detamore MS (2013) Incorporation of aggrecan in interpenetrating network hydrogels to improve cellular performance for cartilage tissue engineering. Tissue Eng Part A 19: 1349-1359.

Jin R, Moreira Teixeira LS, Dijkstra PJ, van Blitterswijk CA, Karperien M, Feijen J (2011) Chondrogenesis in injectable enzymatically crosslinked heparin/dextran hydrogels. J Control Release 152: 186-195.
Jung H, Park JS, Yeom J, Selvapalam N, Park KM, Oh K, Yang JA, Park KH, Hahn SK, Kim K (2014) 3D tissue engineered supramolecular hydrogels for controlled chondrogenesis of human mesenchymal stem cells. Biomacromolecules 15: 707-714.

Katzbauer B (1998) Properties and applications of xanthan gum. Polym Degrad Stab 59: 81-84.

Kesti M, Muller M, Becher J, Schnabelrauch M, D’Este M, Eglin D, Zenobi-Wong M (2015) A versatile bioink for three-dimensional printing of cellular scaffolds based on thermally and photo-triggered tandem gelation. Acta Biomater 11: 162-172.

Kim IL, Khetan S, Baker BM, Chen CS, Burdick JA (2013) Fibrous hyaluronic acid hydrogels that direct MSC chondrogenesis through mechanical and adhesive cues. Biomaterials 34: 5571-5580.

Kim IL, Pfeifer CG, Fisher MB, Saxena V, Meloni GR, Kwon MY, Kim M, Steinberg DR, Mauck RL, Burdick JA (2015) Fibrous scaffolds with varied fiber chemistry and growth factor delivery promote repair in a porcine cartilage defect model. Tissue Eng Part A 21: 2680-2690.

Kitamura N, Yokota M, Kurokawa T, Gong JP, Yasuda $\mathrm{K}$ (2016) In vivo cartilage regeneration induced by a double-network hydrogel: evaluation of a novel therapeutic strategy for femoral articular cartilage defects in a sheep model. J Biomed Mater Res A 104: 2159-2165

Klouda L, Mikos AG (2008) Thermoresponsive hydrogels in biomedical applications. Eur J Pharm Biopharm 68: 34-45.

Kobayashi M, Oka M (2004) Characterisation of a polyvinyl alcohol-hydrogel artificial articular cartilage prepared by injection molding. J Biomater Sci Polym Ed 15: 741-751.

Le NN, Zorn S, Schmitt SK, Gopalan P, Murphy WL (2016) Hydrogel arrays formed via differential wettability patterning enable combinatorial screening of stem cell behavior. Acta Biomater 34: 93-103.

Levett PA, Hutmacher DW, Malda J, Klein TJ (2014) Hyaluronic acid enhances the mechanical properties of tissue-engineered cartilage constructs. PloS One 9: e113216.

Liao IC, Moutos FT, Estes BT, Zhao X, Guilak F (2013) Composite three-dimensional woven scaffolds with interpenetrating network hydrogels to create functional synthetic articular cartilage. Adv Funct Mater 23: 58335839.

Lim JJ, Hammoudi TM, Bratt-Leal AM, Hamilton SK, Kepple KL, Bloodworth NC, McDevitt TC, Temenoff JS (2011) Development of nano- and microscale chondroitin sulphate particles for controlled growth factor delivery. Acta Biomater 7: 986-995.

Lim JJ, Temenoff JS (2013) The effect of desulfation of chondroitin sulfate on interactions with positively charged growth factors and upregulation of cartilaginous markers in encapsulated MSCs. Biomaterials 34: 5007-5018.

Lindahl U, Hook M (1978) Glycosaminoglycans and their binding to biological macromolecules. Annu Rev Biochem 47: 385-417.

Little CJ, Kulyk WM, Chen X (2014) The effect of chondroitin sulphate and hyaluronic acid on chondrocytes 
cultured within a fibrin-alginate hydrogel. J Funct Biomater 5: 197-210.

Lu Z, Doulabi BZ, Huang C, Bank RA, Helder MN (2010) Collagen type II enhances chondrogenesis in adipose tissue-derived stem cells by affecting cell shape. Tissue Eng Part A 16: 81-90.

Makris EA, Gomoll AH, Malizos KN, Hu JC, Athanasiou KA (2015) Repair and tissue engineering techniques for articular cartilage. Nat Rev Rheumatol 11: 21-34.

Marklein RA, Soranno DE, Burdick JA (2012) Magnitude and presentation of mechanical signals influence adult stem cell behavior in 3-dimensional macroporous hydrogels. Soft Matter 8: 8113-8120.

Markstedt K, Mantas A, Tournier I, Avila HM, Hagg D, Gatenholm P (2015) 3D bioprinting human chondrocytes with nanocellulose-alginate bioink for cartilage tissue engineering applications. Biomacromolecules 16: 14891496.

Mendes AC, Baran ET, Pereira RC, Azevedo HS, Reis RL (2012) Encapsulation and survival of a chondrocyte cell line within xanthan gum derivative. Macromol Biosci 12: 350-359.

Moreira Teixeira LS, Bijl S, Pully VV, Otto C, Jin R, Feijen J, van Blitterswijk CA, Dijkstra PJ, Karperien M (2012) Self-attaching and cell-attracting in-situ forming dextran-tyramine conjugates hydrogels for arthroscopic cartilage repair. Biomaterials 33: 3164-3174.

Muller M, Becher J, Schnabelrauch M, Zenobi-Wong M (2015) Nanostructured pluronic hydrogels as bioinks for 3D bioprinting. Biofabrication 7: 035006.

Nehrer S, Breinan HA, Ramappa A, Young G, Shortkroff S, Louie LK, Sledge CB, Yannas IV, Spector M (1997) Matrix collagen type and pore size influence behaviour of seeded canine chondrocytes. Biomaterials 18: $769-776$.

Nguyen LH, Kudva AK, Saxena NS, Roy K (2011) Engineering articular cartilage with spatially-varying matrix composition and mechanical properties from a single stem cell population using a multi-layered hydrogel. Biomaterials 32: 6946-6952.

Oberlender SA, Tuan RS (1994) Spatiotemporal profile of $\mathrm{N}$-cadherin expression in the developing limb mesenchyme. Cell Adhes Commun 2: 521-537.

Ogawa M, Kitamura N, Kurokawa T, Arakaki K, Tanaka Y, Gong JP, Yasuda K (2012) Poly(2-acrylamido-2methylpropanesulfonic acid) gel induces articular cartilage regeneration in vivo: comparisons of the induction ability between single- and double-network gels. J Biomed Mater Res A 100: 2244-2251.

Palumbo FS, Fiorica C, Di Stefano M, Pitarresi G, Gulino A, Agnello S, Giammona G (2015) In situ forming hydrogels of hyaluronic acid and inulin derivatives for cartilage regeneration. Carbohydr Polym 122: 408-416.

Park H, Choi B, Hu J, Lee M (2013) Injectable chitosan hyaluronic acid hydrogels for cartilage tissue engineering. Acta Biomater 9: 4779-4786.

Peterson L, Vasiliadis HS, Brittberg M, Lindahl A (2010) Autologous chondrocyte implantation: a long-term follow-up. Am J Sports Med 38: 1117-1124.
Pirinen S, Karvinen J, Tiitu V, Suvanto M, Pakkanen TT (2015) Control of swelling properties of polyvinyl alcohol/hyaluronic acid hydrogels for the encapsulation of chondrocyte cells. J Appl Polym Sci 132: 42272.

Place ES, Nair R, Chia HN, Szulgit G, Lim EH, Stevens MM (2012) Latent TGF-beta hydrogels for cartilage tissue engineering. Adv Healthc Mater 1: 480-484.

Re'em T, Kaminer-Israeli Y, Ruvinov E, Cohen S (2012) Chondrogenesis of hMSC in affinity-bound TGFbeta scaffolds. Biomaterials 33: 751-761.

Reyes R, Delgado A, Solis R, Sanchez E, Hernandez A, San Roman J, Evora C (2013a) Cartilage repair by local delivery of TGF-beta1 or BMP-2 from a novel, segmented polyurethane/polylactic-co-glycolic bilayered scaffold. J Biomed Mater Res A 102:1110-1120.

Reyes R, Pec MK, Sanchez E, del Rosario C, Delgado A, Evora C (2013b) Comparative, osteochondral defect repair: stem cells versus chondrocytes versus bone morphogenetic protein-2, solely or in combination. Eur Cells Mater 25: 351-365.

Rodell CB, Dusaj NN, Highley CB, Burdick JA (2016) Injectable and cytocompatible tough double-network hydrogels through tandem supramolecular and covalent crosslinking. Adv Mater 28: 8419-8424.

Rodell CB, Kaminski AL, Burdick JA (2013) Rational design of network properties in guest-host assembled and shear-thinning hyaluronic acid hydrogels. Biomacromolecules 14: 4125-4134.

Salinas CN, Anseth KS (2008) The enhancement of chondrogenic differentiation of human mesenchymal stem cells by enzymatically regulated RGD functionalities. Biomaterials 29: 2370-2377.

Schuurman W, Levett PA, Pot MW, van Weeren PR, Dhert WJA, Hutmacher DW, Melchels FPW, Klein TJ, Malda J (2013) Gelatin-methacrylamide hydrogels as potential biomaterials for fabrication of tissue-engineered cartilage constructs. Macromol Biosci 13: 551-561.

Seelbach RJ, Fransen P, Pulido D, D'Este M, Duttenhoefer F, Sauerbier S, Freiman T, Niemeyer P, Albericio F, Alini M, Royo M, Mata A, Eglin D (2015) Injectable hyaluronan hydrogels with peptide-binding dendrimers modulate the controlled release of BMP-2 and TGF-beta 1. Macromol Biosci 15: 1035-1044.

Shi M, Zhu J, Wang R, Chen X, Mi L, Walz T, Springer TA (2011) Latent TGF-beta structure and activation. Nature 474: 343-349.

Simon-Gracia L, Pulido D, Sevrin C, Grandfils C, Albericio F, Royo M (2013) Biocompatible, multifunctional, and well-defined OEG-based dendritic platforms for biomedical applications. Org Biomol Chem 11: 4109-4121.

Singh P, Schwarzbauer JE (2012) Fibronectin and stem cell differentiation - lessons from chondrogenesis. J Cell Sci 125: 3703-3712.

Siu RK, Lu SS, Li W, Whang J, McNeill G, Zhang X, Wu BM, Turner AS, Seim HB, Hoang P, Wang JC, Gertzman AA, Ting K, Soo C (2011) Nell-1 Protein Promotes Bone Formation in a Sheep Spinal Fusion Model. Tissue Eng Part A 17: 1123-1135.

Siu RK, Zara JN, Hou Y, James AW, Kwak J, Zhang X, Ting K, Wu BM, Soo C, Lee M (2012) NELL-1 promotes 
cartilage regeneration in an in vivo rabbit model. Tissue Eng Part A 18: 252-261.

Skaalure SC, Dimson SO, Pennington AM, Bryant SJ (2014) Semi-interpenetrating networks of hyaluronic acid in degradable PEG hydrogels for cartilage tissue engineering. Acta Biomater 10: 3409-3420.

Snyder TN, Madhavan K, Intrator M, Dregalla RC, Park D (2014) A fibrin/hyaluronic acid hydrogel for the delivery of mesenchymal stem cells and potential for articular cartilage repair. J Biol Eng 8: 10.

Spiller KL, Liu Y, Holloway JL, Maher SA, Cao YL, Liu W, Zhou GD, Lowman AM (2012) A novel method for the direct fabrication of growth factor-loaded microspheres within porous nondegradable hydrogels: controlled release for cartilage tissue engineering. J Control Release 157: 39-45.

Spiller KL, Maher SA, Lowman AM (2011) Hydrogels for the repair of articular cartilage defects. Tissue Eng Part B Rev 17: 281-299.

Stammen JA, Williams S, Ku DN, Guldberg RE (2001) Mechanical properties of a novel PVA hydrogel in shear and unconfined compression. Biomaterials 22: 799-806.

Sun AX, Lin H, Beck AM, Kilroy EJ, Tuan RS (2015) Projection stereolithographic fabrication of human adipose stem cell-incorporated biodegradable scaffolds for cartilage tissue engineering. Front Bioeng Biotechnol 3: 115.

van Beuningen HM, Glansbeek HL, van der Kraan PM, van den Berg WB (2000) Osteoarthritis-like changes in the murine knee joint resulting from intra-articular transforming growth factor-beta injections. Osteoarthritis Cartilage 8: 25-33.

Vega SL, Kwon M, Mauck RL, Burdick JA (2016) Single cell imaging to probe Mesenchymal Stem Cell $\mathrm{N}$-cadherin mediated signalling within hydrogels. Ann Biomed Eng 44: 1921-1930.

Vegas AJ, Veiseh O, Doloff JC, Ma M, Tam HH, Bratlie K, Li J, Bader AR, Langan E, Olejnik K, Fenton P, Kang JW, Hollister-Locke J, Bochenek MA, Chiu A, Siebert S, Tang K, Jhunjhunwala S, Aresta-Dasilva S, Dholakia N, Thakrar R, Vietti T, Chen M, Cohen J, Siniakowicz K, Qi M, McGarrigle J, Lyle S, Harlan DM, Greiner DL, Oberholzer J, Weir GC, Langer R, Anderson DG (2016) Combinatorial hydrogel library enables identification of materials that mitigate the foreign body response in primates. Nature Biotechnol 34: 345-352.

Vunjak-Novakovic G, Freed LE (1998) Culture of organized cell communities. Adv Drug Deliv Rev 33: 15 30 .

Wei KC, Zhu ML, Sun YX, Xu JB, Feng Q, Lin S, Wu TY, Xu J, Tian F, Xia J, Li G, Bian LM (2016) Robust biopolymeric supramolecular "host-guest macromer" hydrogels reinforced by in situ formed multivalent nanoclusters for cartilage regeneration. Macromol 49: 866-875.

Whitmire RE, Wilson DS, Singh A, Levenston ME, Murthy N, Garcia AJ (2012) Self-assembling nanoparticles for intra-articular delivery of anti-inflammatory proteins. Biomaterials 33: 7665-7675.

Wong M, Carter DR (2003) Articular cartilage functional histomorphology and mechanobiology: a research perspective. Bone 33: 1-13.
Xu T, Binder KW, Albanna MZ, Dice D, Zhao WX, Yoo JJ, Atala A (2013) Hybrid printing of mechanically and biologically improved constructs for cartilage tissue engineering applications. Biofabrication 5: 015001.

Yasuda K, Kitamura N, Gong JP, Arakaki K, Kwon HJ, Onodera S, Chen YM, Kurokawa T, Kanaya F, Ohmiya Y, Osada Y (2009) A novel double-network hydrogel induces spontaneous articular cartilage regeneration in vivo in a large osteochondral defect. Macromol Biosci 9: 307-316.

Zant E, Grijpma DW (2016a) Synthetic biodegradable hydrogels with excellent mechanical properties and good cell adhesion characteristics obtained by the combinatorial synthesis of photo-cross-linked networks. Biomacromolecules 17: 1582-1592.

Zant E, Grijpma DW (2016b) Tough biodegradable mixed-macromer networks and hydrogels by photocrosslinking in solution. Acta Biomater 31: 80-88.

\section{Discussion with Reviewers}

Dirk Grijpma: Would other polymer systems, such as porous rubber-like materials be suited for cartilage tissue engineering? If not, why would the use of hydrogels be preferred?

Authors: Porous rubber-like materials do present their share of interesting properties and potential advantages to this application, including mechanical properties and resistance to load. However, they are limited in their use for the encapsulation of cells; additionally, injection of such macroporous constructs is difficult, limiting non-invasive implantation techniques. Hydrogels can be used for the encapsulation of cells with uniform distribution and can be injected into irregular defects. Hydrogels also permit nutrient and soluble factor diffusion and in developing tough systems are able to attain mechanical properties comparable to rubber-like materials.

Dirk Grijpma: What would the ideal degradation behaviour of cartilage tissue engineering scaffolds be? And how could that be achieved?

Authors: In principle, the degradation of scaffolds should match or correlate with the formation of matrix by encapsulated or infiltrating cells in the material, which is arguably determined by the differentiation state or phenotype of said cell population. Thus, it is difficult to state a specific timeframe to permit and not inhibit matrix elaboration. Degradation can be tuned through numerous means, including the introduction of hydrolytically degradable crosslinkers or through crosslinkers that degrade in the presence of cell-produced proteases.

Matteo D' Este: Can the authors envisage double network hydrogels based on degradable and cell-instructive (bio) polymers? Could these biodegradable double networks be featured by high toughness but at the same time being capable of cell encapsulation or attraction from surrounding tissues and be used for cartilage regeneration rather than replacement?

Authors: These are great considerations towards the design of hydrogel-based repair for cartilage. Indeed, 
double networks permit tougher hydrogels, and this, in tandem with cell-instructive biomimetic polymers, could promote cartilage regeneration. Some recent works from Jos Malda's and Travis Klein's laboratories did just this, and this has been included in this review. Other work on tough double networks using cell-instructive biopolymers includes a study from Rodell et al. (2016) with hyaluronic acid as the base material, where the material can be both injected and used for cell encapsulation, but to date this has not yet been applied specifically to cartilage tissue engineering.

Dimitrios Zeugolis: Is there a clear hydrogel formulation/ cargo that "leads the race"?

Authors: While we would be interested in identifying a hydrogel formulation that is the best, it is quite difficult to make a specific claim due to the wide variety of experimental parameters explored within the various reports, such as the time points selected or the outcomes measured. While a few studies have compared different hydrogels, none to date are broad or controlled enough to enable us to confidently argue for a specific formulation. Also, only select hydrogels have progressed to clinically relevant animal models and success may depend on the injury model chosen. Furthermore, the best hydrogel will depend on the specific clinical scenario (e.g. patient age, size of defect for repair) and treatment parameters, such as whether cells are delivered or recruited to the tissue site.

Editor's note: The Scientific Editor responsible for this paper was Mauro Alini. 